\title{
The analysis of the generalized square of opposition*
}

\author{
Petra Murinová, Vilém Novák \\ Centre of Excellence IT4Innovations, Division of the University of Ostrava \\ Institute for Research and Applications of Fuzzy Modeling \\ 30. dubna 22, 70200 Ostrava, Czech Republic
}

\begin{abstract}
In this paper, we continue the development of a formal theory of intermediate quantifiers (linguistic expressions such as "most", "many", "few", "almost all", etc.). In previous work, we demonstrated that 105 generalized syllogisms are valid in our theory. We turn our attention to another problem which is the analysis of the generalized Aristotelian square of opposition which, besides the classical quantifiers, is extended also by several selected intermediate quantifiers. We show that the expected relations can be well modeled in our theory. The formal theory of intermediate quantifiers is developed within a special higher-order fuzzy logic — Eukasiewicz fuzzy type theory.
\end{abstract}

Keywords: Fuzzy type theory; Intermediate quantifiers; Aristotelian square of opposition; Complete square of opposition

\section{Introduction}

In this paper, we continue the development of the formal theory of intermediate quantifiers (linguistic expressions such as "most", "many", "few", "almost all", etc.) which was initiated by V. Novák in [7] and continued in [3]. This theory has been inspired by the book of P. L. Peterson [10] and is an attempt to formalize some of its results using an appropriate formal logical system.

Our main idea consists in the assumption that intermediate quantifiers are just classical quantifiers $\forall$ or $\exists$ whose universe of quantification is modified using an evaluative linguistic expression (expression such as "very small", "extremely large", "roughly big", "more or less medium", etc.). Because the meaning of the latter is imprecise, the meaning of intermediate quantifiers is imprecise as well.

The formal theory of intermediate quantifiers is developed using a higher-order fuzzy logic, namely Łukasiewicz fuzzy type theory (denoted by Ł-FTT). Recall that the fuzzy type theory was introduced by V. Novák in [5], first for $\mathrm{IMTL}_{\Delta}$-algebra of truth values and later on also for other kinds of algebras (cf. [8]). The algebra of truth values in E-FTT is a linearly ordered $\mathrm{MV}_{\Delta}$-algebra. Let us emphasize that such kind of the fuzzy logic is strong enough to prove nice properties of our theory.

A constituent of the formal theory of intermediate quantifiers introduced below is also the formal theory of evaluative linguistic expressions. The latter are special expressions of naturale language, for example, small, medium, big, very short quite roughly strong. In this paper, we will consider only simple evaluative expressions with the following syntactical structure:

$$
\langle\text { linguistic hedge }\rangle\langle\text { TE-adjective }\rangle
$$

where 〈TE-adjective〉 is an evaluative adjective (good, interesting, etc.), gradable adjective (small, warm, etc.), and possibly also other specific kind of adjective sharing the following characteristics: TEadjectives semantically characterize various positions on a bounded ordered scale (possibly abstract) and typically form pairs of antonyms (e.g., smallbig) completed by the middle member (medium). Canonical TE-adjectives are small, medium, big.

The 〈linguistic hedge $\rangle$ is an intensifying adverb making the meaning of the evaluative expression either more, or less specific. We distinguish the following:

$$
\begin{aligned}
& \langle\text { linguistic hedge }\rangle:=\text { empty hedge } \mid \\
& \langle\text { narrowing hedge }\rangle \mid\langle\text { widening hedge }\rangle \mid \\
& \langle\text { specifying hedge }\rangle .
\end{aligned}
$$

Typical examples of the linguistic hedges are $e x-$ tremely, significantly, very (narrowing), more or less, roughly, quite roughly, very roughly (widening) or rather (specifying). The empty hedge makes it possible to develop a unified theory of the meaning of an evaluative linguistic expressions by considering the expression "small" as an evaluative expression of the form "empty hedge small" (similarly also "medium" and "big"). We argue that this is correct since there is no essential difference in the meaning of, e.g., "large" and "very large", and other similar expressions.

\footnotetext{
*The paper has been supported by the European Regional Development Fund in the IT4Innovations Centre of Excellence project (CZ.1.05/1.1.00/02.0070).
} 

ions

A special case are the negative evaluative expresnot (empty hedge $\langle$ TE-adjective $\rangle)$.

The meaning of evaluative expressions is formalized within a special theory of E-FTT denoted by $T^{\mathrm{Ev}}$. Semantics of each evaluative expression is in $T^{\mathrm{Ev}}$ construed by a special formula representing an intension whose an interpretation in every model is a function from the set of possible worlds (in our theory, we prefer to speak about contexts) into a set of fuzzy sets. Intension determines in each context the corresponding extension that is a fuzzy set in a certain universe. The extension is constructed as a certain horizon that can be deformed and shifted along the universe. All the details of the formal theory $T^{\mathrm{Ev}}$ including its special axioms and the motivation can be found in [6].

The language of $T^{\mathrm{Ev}}$ contains besides the standard constants $T, \perp$ (truth falsity) also a constant $\dagger \in$ Form $_{o}$ which represents a middle truth value (in the standard Łukasiewicz $\mathrm{MV}_{\Delta}$-algebra, it is interpreted by 0.5 ). Another special constant is $\sim$ (a formula of type $(o o) o$ ) for an additional fuzzy equality on the set of truth values $L$. The theory $T^{\mathrm{Ev}}$ has 11 special axioms which characterize properties of both constants, properties of contexts (see below) and properties of special formulas which represent linguistic hedges.

By the context in $T^{\mathrm{Ev}}$, we understand a formula $w_{\alpha o}$ whose interpretation is a function $w: L \longrightarrow$ $M_{\alpha}$. Hence, the context determines in $M_{\alpha}$ a triple of elements $\left\langle v_{L}, v_{S}, v_{R}\right\rangle$ where $v_{L}, v_{S}, v_{R} \in M_{\alpha}$ and $v_{L}=\mathcal{M}_{p}(w \perp), v_{S}=\mathcal{M}_{p}(w \dagger), v_{R}=\mathcal{M}_{p}(w \top)$.

We introduce the following special linguistic hedges: $\quad\{E x, S i, V e, M L, R o, Q R, V R\}$ (extremely, significantly, very, more or less, roughly, quite roughly, very roughly, respectively) which are ordered as follows:

$$
E x \preceq S i \preceq V e \preceq \bar{\nu} \preceq M L \preceq R o \preceq Q R \preceq V R
$$

By $\preceq$ we denote a relation of a partial ordering of hedges. It can be found in $[6$, p.23]. $\bar{\nu}$ is the empty hedge. Note that the hedges $E x, S i$, Ve have a narrowing effect and $M L, R o, Q R, V R$ have widening effect with respect to the empty hedge.

Specific role in our theory is played by the formulas $\operatorname{Sm} \boldsymbol{\Delta}, \mathrm{Me} \boldsymbol{\Delta}, \mathrm{Bi} \boldsymbol{\Delta}$ where the connective $\boldsymbol{\Delta}$ has been used as a specific hedge that can be taken as a linguistic hedge "utmost" (or, alternatively a "limit"). This makes it possible to include in our theory also classical quantifiers without necessity to introduce them as special cases different from the rest of the theory. How we can see later, the formula $B i \boldsymbol{\Delta}$ will be used for the definitions of the classical quantifiers $\forall$ and $\exists$.

\section{The theory of intermediate quantifiers}

We will introduce a formal theory of intermediate quantifiers $T^{\mathrm{IQ}}$ which is a special theory of E-FTT extending the theory $T^{\mathrm{Ev}}$ of evaluative linguistic expressions introduced in the previous section.

\section{Definition 1}

Let $R \in$ Form $_{o(o \alpha)(o \alpha)}$ be a formula. Put

$$
\mu:=\lambda z_{o \alpha} \lambda x_{o \alpha}\left(R z_{o \alpha}\right) x_{o \alpha} .
$$

We say that the formula $\mu \in$ Form $_{o(o \alpha)(o \alpha)}$ represents a measure on fuzzy sets in the universe of type $\alpha \in$ Types if it has the following properties:

(M1) $\boldsymbol{\Delta}\left(x_{o \alpha} \equiv z_{o \alpha}\right) \equiv\left(\left(\mu z_{o \alpha}\right) x_{o \alpha} \equiv \top\right)$,

(M2) $\boldsymbol{\Delta}\left(x_{o \alpha} \subseteq z_{o \alpha}\right) \& \Delta\left(y_{o \alpha} \subseteq z_{o \alpha}\right) \& \Delta\left(x_{o \alpha} \subseteq\right.$ $\left.y_{o \alpha}\right) \Rightarrow\left(\left(\mu z_{o \alpha}\right) x_{o \alpha} \Rightarrow\left(\mu z_{o \alpha}\right) y_{o \alpha}\right)$,

(M3) $\boldsymbol{\Delta}\left(z_{o \alpha} \quad \not \quad \emptyset_{o \alpha}\right) \& \boldsymbol{\Delta}\left(x_{o \alpha} \subseteq z_{o \alpha}\right) \quad \Rightarrow$ $\left(\left(\mu z_{o \alpha}\right)\left(z_{o \alpha}-x_{o \alpha}\right) \equiv \neg\left(\mu z_{o \alpha}\right) x_{o \alpha}\right)$,

(M4) $\boldsymbol{\Delta}\left(x_{o \alpha} \subseteq y_{o \alpha}\right) \boldsymbol{\&} \boldsymbol{\Delta}\left(x_{o \alpha} \subseteq z_{o \alpha}\right) \boldsymbol{\&} \boldsymbol{\Delta}\left(y_{o \alpha} \subseteq\right.$ $\left.z_{o \alpha}\right) \Rightarrow\left(\left(\mu z_{o \alpha}\right) x_{o \alpha} \Rightarrow\left(\mu y_{o \alpha}\right) x_{o \alpha}\right)$.

One can see that the measure is normed with respect to a distinguished fuzzy set $z_{o \alpha}$.

For the following definition, we have to consider a set of selected types $\mathcal{S}$ to which our theory will be confined. The reason is to avoid possible difficulties with the interpretation of the formula $\mu$ for complex types which may correspond to sets of very large, possibly non-measurable cardinalities. This means that our theory is not fully general. We do not see it as a limitation, though, because one can hardly imagine the meaning of "most $X$ 's" over a set of inaccessible cardinality. On the other hand, our theory works whenever there is a model in which we can define a measure in the sense of Definition 1 . The theory $T^{\mathrm{IQ}}$ defined below is thus parametrized by the set $\mathcal{S}$.

Let us introduce the following special formula representing a fuzzy set of all measurable fuzzy sets in the given type $\alpha$ :

$$
\begin{array}{r}
\mathbf{M}_{o(o \alpha)}:=\lambda z_{o \alpha} \cdot \Delta\left(\mu z_{o \alpha}\right) z_{o \alpha} \&\left(\forall x_{o \alpha}\right)\left(\forall y_{o \alpha}\right) \\
((\mathrm{M} 2) \&(\mathrm{M} 4)) \&\left(\forall x_{o \alpha}\right)(\mathrm{M} 3)
\end{array}
$$

where (M2)-(M4) are the respective axioms from Definition 1.

\section{Definition 2}

Let $\mathcal{S} \subseteq$ Types be a distinguished set of types and $\left\{R \in\right.$ Form $\left._{o(o \alpha)(o \alpha)} \mid \alpha \in \mathcal{S}\right\}$ be a set of new constants. The theory of intermediate quantifiers $T^{I Q}[\mathcal{S}]$ w.r.t. $\mathcal{S}$ is a formal theory of $E-F T T$ defined as follows:

- The language of $T^{I Q}[\mathcal{S}]$ is

$$
J^{E_{V}} \cup\left\{R_{o(o \alpha)(o \alpha)} \in \text { Form }_{o(o \alpha)(o \alpha)} \mid \alpha \in \mathcal{S}\right\} .
$$

- Special axioms of $T^{I Q}[\mathcal{S}]$ are those of $T^{E_{V}}$ and

$$
\left(\exists z_{o \alpha}\right) \mathbf{M}_{o(o \alpha)} z_{o \alpha}, \quad \alpha \in \mathcal{S} .
$$

Intermediate quantifiers have been formally defined by Novák in [7]. The following definition is a slight modification of the original definition by considering strong conjunction instead of the ordinary one. 


\section{Definition 3}

Let $T^{I Q}[\mathcal{S}]$ be a theory of intermediate quantifiers in the sense of Definition 2 and $E v \in$ Form $_{o o}$ be intension of some evaluative expression. Furthermore, let $z \in$ Form $_{o \alpha}, x \in$ Form $_{\alpha}$ be variables and $A, B \in$ Form $_{o \alpha}$ be formulas, $\alpha \in \mathcal{S}$, such that

$$
T^{I Q} \vdash \mathbf{M}_{o(o \alpha)} B_{o \alpha}
$$

holds true. Then a type $\langle 1,1\rangle$ intermediate generalized quantifier interpreting the sentence

" $\langle$ Quantifier $\rangle$ B's are A"

is one of the following formulas:

$$
\begin{gathered}
\left(Q_{E v}^{\forall} x\right)(B, A):= \\
(\exists z)((\boldsymbol{\Delta}(z \subseteq B) \&(\forall x)(z x \Rightarrow A x)) \wedge E v((\mu B) z)),
\end{gathered}
$$

$$
\begin{aligned}
& \left(Q_{E v}^{\exists} x\right)(B, A):= \\
& (\exists z)((\Delta(z \subseteq B) \&(\exists x)(z x \wedge A x)) \wedge E v((\mu B) z)) .
\end{aligned}
$$

These quantifiers represent formal meaning of natural language expressions, such as

"most(many, a lot of, a few, some etc.) B's are A".

If the presupposition is necessary then the following definition must be considered.

\section{Definition 4}

Let $T^{I Q}[\mathcal{S}]$ be a theory of intermediate quantifiers in the sense of Definition 2 and $E v, z, x, A, B$ be the same as in Definition 3. Then an intermediate generalized quantifier with presupposition is the formula

$$
\begin{gathered}
\left.{ }^{*} Q_{E v}^{\forall} x\right)(B, A) \equiv(\exists z)((\Delta(z \subseteq B) \&(\exists x) z x \& \\
(\forall x)(z x \Rightarrow A x)) \wedge E v((\mu B) z)) .
\end{gathered}
$$

Note that only non-empty subsets of $B$ are considered in this definition.

We continue with the theorem which says us that the hedge "utmost" degenerates intermediate quantifiers to the ordinary classical ones. Do not forget, that still truth values of the respective formulas can lay between zero and unit.

\section{Theorem 1 ([7])}

The following is equivalent:

- $T^{I Q} \vdash\left(Q_{B i \Delta}^{\forall} x\right)(B, A) \equiv(\forall x)(B x \Rightarrow A x)$,

- $T^{I Q} \vdash\left(Q_{B i \Delta}^{\exists} \Delta\right)(B, A) \equiv(\exists x)(B x \Rightarrow A x)$.

We will now introduce definitions of several specific intermediate quantifiers based on the analysis provided by Peterson in his book [10].

A: All $B$ are $A:=\left(Q_{B i \Delta}^{\forall} x\right)(B, A) \equiv(\forall x)(B x \Rightarrow A x)$,

E: No $B$ are $A:=\left(Q_{B i \Delta}^{\forall} x\right)(B, \neg A) \equiv(\forall x)(B x \Rightarrow \neg A x)$,

P: Almost all $B$ are $A:=\left(Q_{B i E x}^{\forall} x\right)(B, A) \equiv$

$$
(\exists z)((\boldsymbol{\Delta}(z \subseteq B) \boldsymbol{\&}(\forall x)(z x \Rightarrow A x)) \wedge(B i E x)((\mu B) z)),
$$

B: Few $B$ are $A(:=$ Almost all $B$ are not $A):=\left(Q_{B i E x}^{\forall} x\right)(B, \neg A) \equiv$

$$
(\exists z)((\boldsymbol{\Delta}(z \subseteq B) \&(\forall x)(z x \Rightarrow \neg A x)) \wedge(B i E x)((\mu B) z)),
$$

T: Most $B$ are $A:=\left(Q_{B i \text { Ve }}^{\forall} x\right)(B, A) \equiv$

$$
(\exists z)((\boldsymbol{\Delta}(z \subseteq B) \boldsymbol{\&}(\forall x)(z x \Rightarrow A x)) \wedge(B i V e)((\mu B) z)),
$$

D: Most $B$ are not $A:=\left(Q_{B i V e}^{\forall} x\right)(B, \neg A) \equiv$

$$
(\exists z)((\boldsymbol{\Delta}(z \subseteq B) \boldsymbol{\&}(\forall x)(z x \Rightarrow \neg A x)) \wedge(B i V e)((\mu B) z)),
$$

K: Many $B$ are $A:=\left(Q_{\neg(S m \bar{\nu})}^{\forall} x\right)(B, A) \equiv$

$$
(\exists z)((\boldsymbol{\Delta}(z \subseteq B) \boldsymbol{\&}(\forall x)(z x \Rightarrow A x)) \wedge \neg(\operatorname{Sm} \overline{\boldsymbol{\nu}})((\mu B) z)),
$$

G: Many $B$ are not $A:=\left(Q_{\neg(S m \overline{\boldsymbol{\nu}})}^{\forall} x\right)(B, \neg A) \equiv$

$$
(\exists z)((\boldsymbol{\Delta}(z \subseteq B) \&(\forall x)(z x \Rightarrow \neg A x)) \wedge \neg(\operatorname{Sm} \overline{\boldsymbol{\nu}})((\mu B) z)),
$$

I: Some $B$ are $A:=\left(Q_{B i \Delta}^{\exists} x\right)(B, A) \equiv(\exists x)(B x \wedge A x)$,

O: Some $B$ are not $A:=\left(Q_{B i \Delta}^{\exists} x\right)(B, \neg A) \equiv(\exists x)(B x \wedge \neg A x)$.

For example, the quantifier $\mathbf{P}$ says that we consider the greatest fuzzy set $z$ being subset of $B$ such that all its elements have the property $A$ and $z$ is "extremely big" (in the sense of the measure $\mu$ ).
The meaning of the other intermediate quantifiers is similar. Note that the quantifiers $\mathbf{A}, \mathbf{E}, \mathbf{I}, \mathbf{O}$ use $\Delta$ as a hedge and so, they coincide with the classical quantifiers defined in first-order classical logic 
(cf. [7]). Recall that the formula $(B i E x)((\mu B) z)$ represents the truth value of the linguistic expression "the measure $(\mu B) z$ of the fuzzy set $z$ is extremely big", $(S m \overline{\boldsymbol{\nu}})((\mu B) z)$ represents truth value of the linguistic expression "the measure $(\mu B) z$ of the fuzzy set $z$ is small" and similarly the others formulas.

\section{Remark 1}

By ${ }^{*} \mathbf{A},{ }^{*} \mathbf{E},{ }^{*} \mathbf{P},{ }^{*} \mathbf{B},{ }^{*} \mathbf{T},{ }^{*} \mathbf{D},{ }^{*} \mathbf{K},{ }^{*} \mathbf{G}$ we denote quantifiers which contain the presupposition. Analogously, the specific quantifiers "Most", "Many", etc. with the presupposition will be written as "*Most", "*Many", etc.

\section{Remark 2}

Let us emphasize that there are two meanings of the quantifier "Most", namely "more than half" and "close to all". In our theory, we construe "Most" in the second meaning.

The following theorem demonstrates that monotonicity plays an important role in our theory.

\section{Theorem 2}

Let $\boldsymbol{A}, \ldots, \boldsymbol{G}$ be the basic intermediate quantifiers defined above. Then the following sets of implications are provable in $T^{I Q}$ :

$$
\begin{array}{ll}
\text { - } T^{I Q} \vdash \mathbf{A} \Rightarrow \mathbf{P}, & T^{I Q} \vdash \mathbf{P} \Rightarrow \mathbf{T}, \\
& T^{I Q} \vdash \mathbf{T} \Rightarrow \mathbf{K} . \\
\text { - } & T^{I Q} \vdash \mathbf{E} \Rightarrow \mathbf{B}, \quad T^{I Q} \vdash \mathbf{B} \Rightarrow \mathbf{D}, \\
& T^{I Q} \vdash \mathbf{D} \Rightarrow \mathbf{G} .
\end{array}
$$

If the presupposition is needed then also the following theorem is provable.

\section{Theorem 3}

Let $\boldsymbol{A}, \ldots, \boldsymbol{G}$ be the basic intermediate quantifiers defined above. Then the following sets of implications are provable in $T^{I Q}$ :

$$
\begin{aligned}
& \text { - } T^{I Q} \vdash{ }^{*} \mathbf{A} \Rightarrow{ }^{*} \mathbf{P}, \quad T^{I Q} \vdash{ }^{*} \mathbf{P} \Rightarrow{ }^{*} \mathbf{T}, \\
& T^{I Q} \vdash{ }^{*} \mathbf{T} \Rightarrow{ }^{*} \mathbf{K} \text {. } \\
& \text { - } T^{I Q} \vdash{ }^{*} \mathbf{E} \Rightarrow{ }^{*} \mathbf{B}, \quad T^{I Q} \vdash{ }^{*} \mathbf{B} \Rightarrow{ }^{*} \mathbf{D} \text {, } \\
& T^{I Q} \vdash{ }^{*} \mathbf{D} \Rightarrow{ }^{*} \mathbf{G} \text {. }
\end{aligned}
$$

Both theorems say that in arbitrary model, the truth value of the quantifier on the left side is smaller than or equal to the truth value of the quantifiers on the right side. In [3], we constructed a model of $T^{\mathrm{IQ}}$. Consequently, the theory $T^{\mathrm{IQ}}$ is consistent by completeness.

\section{Analysis of the generalized square of opposition}

In this section, we will deal with the main task of this paper which is to study and formally analyze relationships among intermediate quantifiers in a generalized complete square of opposition studied first by Thompson in [12]. There are many publications that are related to this area. Recall the work of
Peterson [10] and many others (see [4, 14]). In [9], the author presents classical and modern squares of opposition, where the problem with presupposition is discussed. Below, we will show how presupposition is analysed in our theory. In [2], the author introduced generalized quantifiers and the classical square of opposition based on first-order classical predicate logic. We can find here definitions of generalized quantifiers as well as definitions of many other relations between generalized quantifiers (equivalence, anti-subalternation, antisuperalternation) that are defined in classical logic. The semantical properties of generalized quantifiers (isomorphism, extension and conservativity) based on classical logic are studied in [1]. All of the cited papers consider classical logic only. Recall that the main idea of this paper is model generalized Aristotle and also Peterson's square of opposition using higher-order fuzzy logic.

The square comprises relations among generalized intermediate quantifiers. Analogously to the classical square, we will also consider relations of contrary, contradictory and subcontrary in $\mathrm{E}$ FTT and show that the generalized complete square formed of the generalized intermediate quantifiers defined above can be constructed also in E-FTT..

\subsection{Formalization of relations among quantifiers in E-FTT}

In this subsection, we denote by $P_{1}, P_{2} \in$ Form $_{o}$ formulas of type $o$ (i.e. propositions).

\section{Definition 5 (Contraries)}

We say that $P_{1}, P_{2}$ are contraries in $T^{I Q}$ if in every model $\mathcal{M} \models T^{I Q}$ the following is true:

$$
\mathcal{M}\left(P_{1}\right) \otimes \mathcal{M}\left(P_{2}\right)=\mathcal{M}(\perp) .
$$

Due to completeness theorem, we can alternatively say that $P_{1}$ and $P_{2}$ are contraries if $T^{\mathrm{IQ}} \vdash$ $P_{1} \& P_{2} \equiv \perp$.

\section{Definition 6 (Sub-contraries)}

We say that $P_{1}$ and $P_{2}$ are sub-contraries in $T^{I Q}$ if in every model $\mathcal{M} \models T^{I Q}$ the following is true:

$$
\mathcal{M}\left(P_{1}\right) \oplus \mathcal{M}\left(P_{2}\right) \neq \mathcal{M}(\perp) .
$$

Analogously as above $P_{1}$ and $P_{2}$ are sub-contraries, if $T^{I Q} \vdash P_{1} \nabla P_{2} \not \equiv \perp$.

The first idea how to define contradictories in $T^{\mathrm{IQ}}$ was to say that $P_{1}$ and $P_{2}$ are contradictories if they are both contraries and subcontraries. Unfortunately, this does not work because the classical quantifiers $\mathbf{A}$ and $\mathbf{O}$, as well as $\mathbf{E}$ and $\mathbf{I}$ must be contradictories. However, if we consider $T^{\mathrm{IQ}}$ and realize that $\vdash \neg(\forall x)(B x \Rightarrow A x) \equiv(\exists x)(B x \boldsymbol{\&} \neg A x)$ then

$$
T^{\mathrm{IQ}} \vdash \neg \mathbf{A} \neq \mathbf{O}
$$

since $\mathbf{O}:=(\exists x)(B x \wedge \neg A x)$. The use of the delta connective solves the problem. 


\section{Definition 7 (Contradictories)}

The formulas $P_{1}$ and $P_{2}$ are contradictories in $T^{I Q}$ if in every model $\mathcal{M} \models T^{I Q}$ the following two equalities hold:

(a) $\mathcal{M}\left(\Delta P_{1}\right) \otimes \mathcal{M}\left(\Delta P_{2}\right)=\mathcal{M}(\perp)$,

(b) $\mathcal{M}\left(\Delta P_{1}\right) \oplus \mathcal{M}\left(\Delta P_{2}\right)=\mathcal{M}(\top)$.

Alternatively we can say that $P_{1}$ and $P_{2}$ are contradictories, if both $T^{I Q} \vdash \Delta P_{1} \& \Delta P_{2} \equiv \perp$ as well as $T^{I Q} \vdash \Delta P_{1} \nabla \Delta P_{2}$.

\section{Definition 8 (Subalterns)}

The formula $P_{1}$ is a subaltern of $P_{2}$ in $T^{I Q}$ if in every model $\mathcal{M} \models T^{I Q}$ the inequality

$$
\mathcal{M}\left(P_{1}\right) \leq \mathcal{M}\left(P_{2}\right)
$$

holds true. We will call $P_{2}$ a superaltern of $P_{1}$. Alternatively we can say that $P_{1}$ is a subaltern of $P_{2}$ if $T^{I Q} \vdash P_{1} \Rightarrow P_{2}$.

\subsection{Aristotle square of opposition in $T^{\mathrm{IQ}}$}

In this section, we will formally prove that in $T^{\mathrm{IQ}}$ the above properties are shared by the classical quantifiers. On many places in formal proofs, we write simply "by properties of Ł-FTT" and refer the reader to the cited papers for the details. We will also fix the set $\mathcal{S}$ and write $T^{\mathrm{IQ}}$ instead of $T^{\mathrm{IQ}}[\mathcal{S}]$.

\section{Theorem 4 (Contraries)}

(a) The formulas ${ }^{*} \mathbf{A}, \mathbf{E}$ are contraries in $T^{I Q}$.

(b) If $T^{I Q} \vdash(\exists x) B x$ then the formulas $\mathbf{A}, \mathbf{E}$ are contraries in $T^{I Q}$.

\section{Theorem 5 (Sub-contraries)}

If $T^{I Q} \vdash(\exists x) B x$ then the formulas $\mathbf{O}$ and $\mathbf{I}$ are sub-contraries in $T^{I Q}$.

\section{Theorem 6 (Contradictories)}

(a) The formulas $\mathbf{A}$ and $\mathbf{O}$ are contradictories in $T^{I Q}$.

(b) The formulas $\mathbf{E}$ and $\mathbf{I}$ are contradictories in $T^{I Q}$.

\section{Theorem 7 (Subalterns)}

(a) The formula ${ }^{*} \mathbf{A}$ is subaltern of $\mathbf{I}$ in $T^{I Q}$.

(b) If $T^{I Q} \vdash(\exists x) B x$, then the formula $\mathbf{A}$ is subaltern of $\mathbf{I}$ in $T^{I Q}$.

(c) The formula $\mathbf{E}$ is subaltern of ${ }^{*} \mathbf{O}$ in $T^{I Q}$.

(d) If $T^{I Q} \vdash(\exists x) B x$, then $\mathbf{E}$ is subaltern of $\mathbf{O}$ in $T^{I Q}$.

We introduce the example of the interpretation of generalized Aristotelian square of opposition in $T^{\mathrm{IQ}}$.

\section{Example 1}

Let us consider a model $\mathcal{M} \models T^{I Q}$ such that $T^{I Q} \vdash$ $(\exists x) B x$ and let $\mathcal{M}(\mathbf{A})=a>0$ (e.g., $a=0.2$ ). Since $\mathbf{A}, \mathbf{E}$ are contraries, we have $\mathcal{M}(\mathbf{E})=e \leq$ $1-a$. Because the formulas $\mathbf{A}$ and $\mathbf{O}$ are contradictories, it follows from the definition of contradictories that $\mathcal{M}(\Delta \mathbf{A})=0$ and so $\mathcal{M}(\Delta \mathbf{O})=1$ because $\mathcal{M}(\boldsymbol{\Delta} \mathbf{A}) \otimes \mathcal{M}(\boldsymbol{\Delta} \mathbf{O})=0$ and $\mathcal{M}(\boldsymbol{\Delta} \mathbf{A}) \oplus \mathcal{M}(\boldsymbol{\Delta} \mathbf{O})=1$. Consequently, $\mathbf{E}$ is subaltern of $\mathbf{O}$.

The $\mathbf{I}$ is superaltern of $\mathbf{A}$ and thus $\mathcal{M}(\mathbf{I})=i \geq$ 0.2 . However, $\mathbf{I}$ is contradictory with $\mathbf{E}$ and so $\mathcal{M}(\mathbf{I})=i=1$. Finally, $\mathbf{I}$ is sub-contrary with $\mathbf{O}$ because $\mathcal{M}(\mathbf{O} \nabla \mathbf{I})=1$ and $\mathbf{I}$ is superaltern of $\mathbf{A}$. By the straight lines mark contradictories, the dashed lines contraries, the dotted lines sub-contraries, and the arrows indicate subalterns.

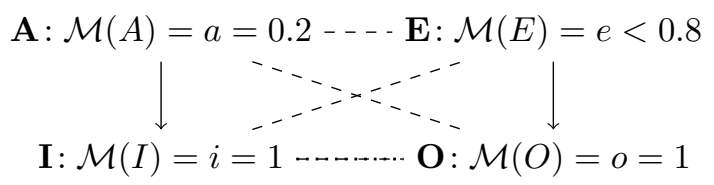

\subsection{Necessary extension of $T^{\mathrm{IQ}}$}

The complete generalized square of opposition contains couples of intermediate quantifiers $\left(Q_{E v}^{\forall} x\right)(B, A)$ and $\left(Q_{E v}^{\forall} x\right)\left(B^{\prime}, \neg A\right)$. The universes $B$ and $B^{\prime}$, however, can be different. To be able to compare both quantifiers, we need the universes $B$ and $B^{\prime}$ to be equal. Additionally, it is quite often also desirable that the universe is a normal fuzzy set. Therefore, we must introduce a new special theory $T\left[B, B^{\prime}\right]$ being a consistent extension of $T^{\mathrm{IQ}}$ in which the above requirements are fulfilled.

\section{Definition 9}

Let $B, B^{\prime} \in$ Form $_{o \alpha}$ be selected formulas. The theory $T\left[B, B^{\prime}\right]$ is a consistent extension of $T^{I Q}$ such that

(a) $T\left[B, B^{\prime}\right] \vdash B \equiv B^{\prime}$,

(b) $T\left[B, B^{\prime}\right] \vdash \quad\left(\exists x_{\alpha}\right) \Delta B x$ and $T\left[B, B^{\prime}\right] \quad \vdash$ $\left(\exists x_{\alpha}\right) \Delta B^{\prime} x$.

The first axiom assures that both universes are the same and the second two axioms assure their normality. If we consider the theory $T\left[B, B^{\prime}\right]$ in the sequel then the formulas $B, B^{\prime}$ will always be taken as universes of quantification of intermediate quantifiers in concern.

\section{Remark 3}

To make the theory of intermediate quantifiers more general, we did not specify explicitly axioms of the theory $T\left[B, B^{\prime}\right]$. Instead, we only require in Definition 10 that $T\left[B, B^{\prime}\right]$ is consistent and (a) and (b) are provable. It is not difficult to construct a model of $T^{I Q}$ in which (a) and (b) are true and so, the simplest consistent extension of $T^{I Q}$ can be obtained by taking (a) and (b) as new special axioms.

We introduce a special theory $T\left[B, B^{\prime}\right]$ which is a consistent extension of $T^{\mathrm{IQ}}$ where $B, B^{\prime}$ are formulas occurring in the definition of special intermediate quantifiers. The reason is that, when studying 
relations of two intermediate generalized quantifiers we need them to be defined on the same universes which, moreover, are normal fuzzy sets. These requirements lead to the following definition.

\section{Definition 10}

Let $B, B^{\prime} \in$ Form $_{o \alpha}$. The theory $T\left[B, B^{\prime}\right]$ is a consistent extension of $T^{I Q}$ such that

(a) $T\left[B, B^{\prime}\right] \vdash B \equiv B^{\prime}$,

(b) $T\left[B, B^{\prime}\right] \vdash\left(\exists x_{\alpha}\right) \Delta B x$,

(c) $T\left[B, B^{\prime}\right] \vdash\left(\exists x_{\alpha}\right) \Delta B^{\prime} x$.

This means, that if we study two intermediate quantifiers (positive and negative) then we always work with the following formulas:

$$
\begin{gathered}
\left(Q_{E v}^{\forall} x\right)(B, A):= \\
(\exists z)((\Delta(z \subseteq B) \&(\forall x)(z x \Rightarrow A x)) \wedge E v((\mu B) z))
\end{gathered}
$$

and

$$
\begin{aligned}
\left(Q_{E v}^{\forall} x\right) & \left(B^{\prime}, A\right):=\left(\exists z^{\prime}\right)\left(\left(\Delta\left(z^{\prime} \subseteq B^{\prime}\right) \&\right.\right. \\
(\forall x)\left(z^{\prime} x\right. & \left.\Rightarrow \neg A x)) \wedge E v\left(\left(\mu B^{\prime}\right) z^{\prime}\right)\right) .
\end{aligned}
$$

Obviously, all relations in Subsection 3.2 hold also in $T\left[B, B^{\prime}\right]$.

\subsection{Properties of intermediate quantifiers in $T\left[B, B^{\prime}\right]$}

This subsection contains formal proofs of properties of the intermediate quantifiers described in the complete square of opposition. The $z \in$ Form $_{o \alpha}$, $x \in$ Form $_{\alpha}$ are variables and $A, B \in$ Form $_{o \alpha}$ are formulas, $\alpha \in \mathcal{S}$.

\section{Theorem 8 (Contraries)}

(a) The formulas $\mathbf{B}$ and $\mathbf{P}$ are contraries in $T\left[B, B^{\prime}\right]$. (b) The formulas $\mathbf{D}$ and $\mathbf{T}$ are contraries in $T\left[B, B^{\prime}\right]$.

\section{Theorem 9 (Contraries)}

The following couples of formulas are contraries in the theory $T\left[B, B^{\prime}\right]$ :
(a) $\mathbf{G}$ and $\mathbf{P}$,
(b) $\mathbf{K}$ and $\mathbf{B}$,
(c) $\mathbf{E}$ and $\mathbf{K}$,
(d) $\mathbf{E}$ and $\mathbf{T}$,
(e) $\mathbf{E}$ and $\mathbf{P}$,
(f) $\mathbf{A}$ and $\mathbf{G}$,
(g) $\mathbf{A}$ and $\mathbf{D}$,
(h) $\mathbf{A}$ and $\mathbf{B}$,
(i) $\mathbf{B}$ and $\mathbf{T}$,
(j) $\mathbf{P}$ and $\mathbf{D}$.

\section{Theorem 10 (Subalterns)}

The following holds in $T\left[B, B^{\prime}\right]$ :
(a) ${ }^{*} \mathbf{A}$ is subaltern of ${ }^{*} \mathbf{P}$,
(b) ${ }^{*} \mathbf{P}$ is subaltern of ${ }^{*} \mathbf{T}$,
(c) ${ }^{*} \mathbf{T}$ is subaltern of ${ }^{*} \mathbf{K}$,
(d) ${ }^{*} \mathbf{K}$ is subaltern of $\mathbf{I}$,
(e) ${ }^{*} \mathbf{E}$ is subaltern of ${ }^{*} \mathbf{B}$,
(f) ${ }^{*} \mathbf{B}$ is subaltern of ${ }^{*} \mathbf{D}$,
(g) ${ }^{*} \mathbf{D}$ is subaltern of ${ }^{*} \mathbf{G}$,
(h) ${ }^{*} \mathbf{G}$ is subaltern of $\mathbf{O}$.

\subsection{Generalized complete square of opposition}

We finish this paper with the figure depicting the generalized complete square with contradictions, contraries, sub-contraries and subalterns which generalizes the classical complete square of opposition. Recall that, the straight lines mark contradictories, the dashed lines contraries, the dotted lines subcontraries, and the arrows indicate subalterns.

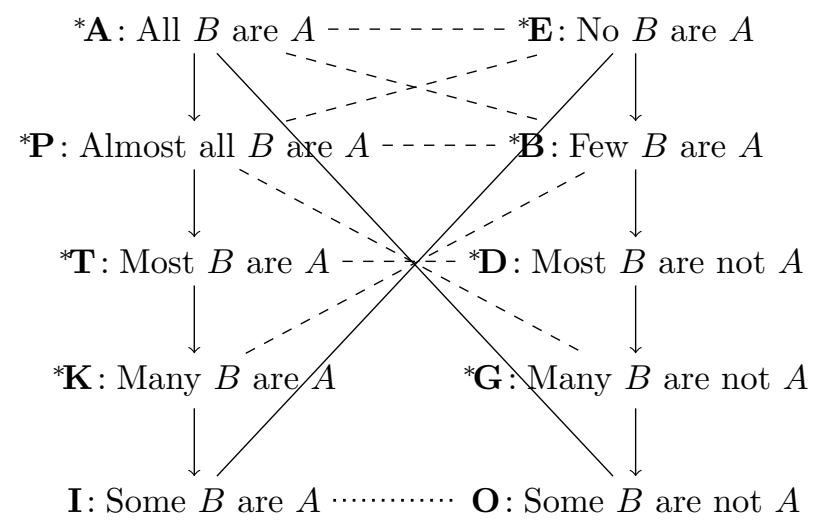

Finally, we review the formulas having different properties in comparison with the classical Peterson's square of opposition. Namely, we showed that the couples formulas ${ }^{*} \mathbf{P}$ and ${ }^{*} \mathbf{G}$, and ${ }^{*} \mathbf{B}$ and ${ }^{*} \mathbf{K}$ are contraries (they are contradictories in the classical complete square).

\section{Remark 4}

In the scheme above, we omitted relations among the quantifiers $\mathbf{K}, \mathbf{O}, \mathbf{G}$ and $\mathbf{I}$. The reason is that the quantifiers $\mathbf{K}$ and $\mathbf{G}$ and "typically fuzzy" in the sense, that their meaning is very indefinite. Therefore, the relations among them and the other quan- 
tifiers are more intricate and depend also on inner relations between the considered subsets of their universe. Consequently, it seems that depending on the specific situation, the relation between $\mathbf{K}$ and $\mathbf{G}$ can vary from contrary to subcontrary. This is not yet well understood and so, we postpone this part of our analysis to a subsequent paper.

\section{Example 2}

Let $\mathcal{M} \models T\left[B, B^{\prime}\right]$ be a model such that $\mathcal{M}\left({ }^{*} \mathbf{P}\right)>0$ (e.g., $0<p \leq 0.4$ ). Let us denote $\mathcal{M}\left({ }^{*} \mathbf{A}\right)=a$, $\mathcal{M}\left({ }^{*} \mathbf{E}\right)=e, \mathcal{M}\left({ }^{*} \mathbf{B}\right)=b, \mathcal{M}\left({ }^{*} \mathbf{T}\right)=t, \mathcal{M}\left({ }^{*} \mathbf{D}\right)=d$, $\mathcal{M}\left({ }^{*} \mathbf{K}\right)=k, \mathcal{M}\left({ }^{*} \mathbf{G}\right)=g, \mathcal{M}(\mathbf{O})=o$ and $\mathcal{M}(\mathbf{I})=i$.

Because the formula ${ }^{*} \mathbf{P}$ is contrary both with ${ }^{*} \mathbf{E}$ as well as with ${ }^{*} \mathbf{B}$, we have $\mathcal{M}\left({ }^{*} \mathbf{B}\right)=b \leq 1-p$ and $\mathcal{M}\left({ }^{*} \mathbf{E}\right)=e \leq 1-p$ where $e \leq b$ because ${ }^{*} \mathbf{E}$ is subaltern of ${ }^{*} \mathbf{B}$.

Since the formula * $\mathbf{A}$ is subaltern of ${ }^{*} \mathbf{P}$, we have $a \leq p$. From the fact that the couples of formulas ${ }^{*} \mathbf{A}$ and $\mathbf{O}$, and ${ }^{*} \mathbf{E}$ and $\mathbf{I}$ are contradictory it follows that $\mathcal{M}(\mathbf{I})=i=1$ and $\mathcal{M}(\mathbf{O})=o=1$. Because the formula ${ }^{*} \mathbf{T}$ is contrary with ${ }^{*} \mathbf{D}$ and ${ }^{*} \mathbf{T}$ is superaltern of ${ }^{*} \mathbf{P}$ then $p \leq t$ where $t \otimes d=0$. We may verify that also $p \otimes d=0$ as well as $b \otimes t=0$ which means that the formulas ${ }^{*} \mathbf{P},{ }^{*} \mathbf{D}$ and ${ }^{*} \mathbf{T},{ }^{*} \mathbf{B}$ are indeed contraries. All the results are summarized in the following picture (recall that by the straight lines mark contradictories, the dashed lines contraries, the dotted lines sub-contraries, and the arrows indicate subalterns):

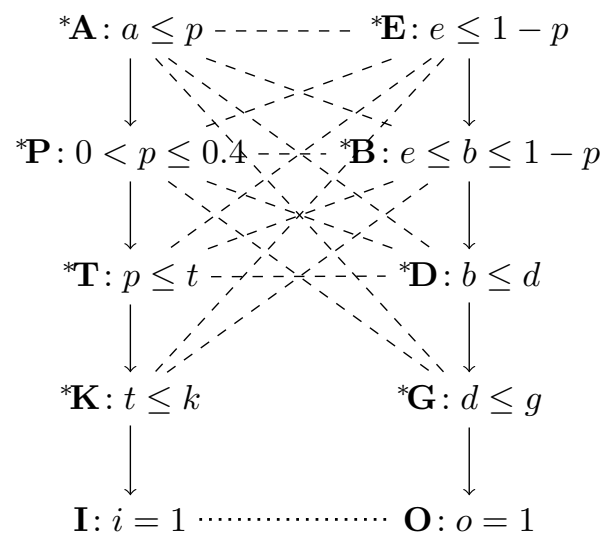

If we set some concrete truth degrees, we obtain the following result:

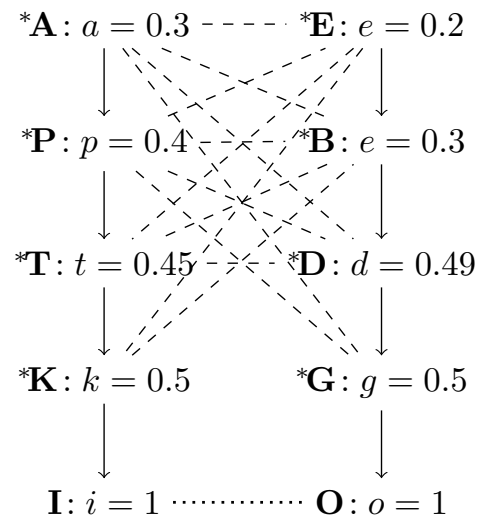

\section{Conclusion}

This paper is a continuation of the formal theory of intermediate quantifiers which are special linguistic expressions such as "most", "many", "few", "almost all", etc. Using means of Łukasiewicz fuzzy type theory we developed their semantics and demonstrated that many relations studied informally in the literature hold also in our theory. Following the analysis of the generalized syllogisms presented in the previous paper [3], we continued here the analysis of generalized Aristotelian square of opposition which contains, besides the classical quantifiers, also selected intermediate ones. We introduced formal definitions of contraries, subcontraries, contradictories and subalterns in our theory and demonstrated that they naturally generalize the classical properties which can thus be extended also to generalized intermediate quantifiers.

The future work will focus on extension of the list of generalized intermediate quantifiers by further specific ones, definition of their semantics and on the analysis of their syllogisms and also properties of the generalized square of opposition.

\section{References}

[1] D'Alfonso, D., The Square of Opposition and Generalized Quantifiers,in: J.Y.Béziau and D. Jacquette (eds), Around and Beyond the Square of Opposition, Studies in Universal Logic, Birkhäuser, Berlin, (2012).

[2] Brown, M., Generalized Quantifiers and the Square of Opposition, Notre Dame Journal of Formal Logic, 25, 303-322, 1984.

[3] P. Murinová and V. Novák, A Formal Theory of Generalized Intermediate Syllogisms, Fuzzy Sets and Systems, 186, 47-80,2012.

[4] Afshar,M. and Dartnell, Ch. and Luzeaux, D. and Sallantin, J. and Tognetti,Y., Aristotle's Square Revisited to Frame Discovery Science, Journal of Computers, 2, 54-66, 2007.

[5] V. Novák, On fuzzy type theory, Fuzzy Sets and Systems, 149, 235-273, 2005.

[6] V. Novák, A comprehensive theory of trichotomous evaluative linguistic expressions, Fuzzy Sets and Systems, 159 (22), 2939-2969, 2008.

[7] V. Novák, A formal theory of intermediate quantifiers, Fuzzy Sets and Systems, 159 (10), 12291256, 2008.

[8] V. Novák, EQ-algebra-based fuzzy type theory and its extension, Fuzzy Sets and Systems, 159 (22), 2939-2969, 2008.

[9] Parsons, T. Things That are Right with the Traditional Square of Opposition, Logica Universalis, 2, 3-11, 2008.

[10] P. P. Peterson. Intermediate quantifiers, Logic, linguistics, Aristotelian semantics, Ahgate, Aldershot, 2000. 
[11] Peters, S. and Westerståhl, D.Quantifiers in Language and Logic, Oxford, Claredon Press, 2006.

[12] B. E. Thompson, Syllogisms using "few", "many" and "most", Notre Dame Journal of Formal Logic, (23), 75-84, 1982.
[13] Westerståhl, D. The traditional square of opposition and generalized quantifiers, Studies in Logic, 2, 1-18, 2008.

[14] Wikipedia, http://en.wikipedia.org/wiki/Aristotle, 2004. 